\title{
Sport injuries in enduro riders: a review of literature
}

\author{
Anil Khanna ${ }^{1}$ \\ Elmunzar O. Bagouri' ${ }^{1}$ \\ Nikolaos Gougoulias ${ }^{1}$ \\ Nicola Maffulli ${ }^{2}$
}

1 Department of Trauma and Orthopaedic Surgery, Keele Universıty School of Medicine, University Hospital of North Staffordshire, Stoke on Trent, England

2 Department of Musculoskeletal Disorders, Faculty of Medicine and Surgery, University of Salerno, Baronissi, Salerno, Italy

\section{Corresponding author:}

Nicola Maffulli

Department of Musculoskeletal Disorders, Faculty of Medicine and Surgery, University of Salerno, Baronissi, Salerno, Italy

Centre for Sports and Exercise Medicine

Barts and The London School of Medicine and Dentistry

Mile End Hospital

275 Bancroft Road

London E1 4DG, England

E-mail: n.maffulli@qmul.ac.uk

\section{Summary}

Introduction: enduro is an off road motorcycling event. It is a fast, exciting adventure sport with increasing numbers of participants and competitions. Materials and methods: we performed search of PubMed, Medline, CINAHL, and Embase databases using the following keywords 'Enduro injuries', 'off-road motorcycle injuries' and 'Enduro sport'. We identified four studies which described the physiological characteristic of enduro riders and the injury pattern sustained by these athletes.

Results: hands, wrists and forearms are the predominant areas of overuse in enduro riders. The extremities are the most injured parts in enduro. However, $98 \%$ of these injuries are mild to moderate with abbreviated injury scale grades 1 and 2 .

Conclusion: there is paucity of published data on enduro injuries. In depth understanding of the physiological aspect of enduro riders with close monitoring of injuries is needed to promote safety measures in enduro and to reduce risk factors of injury which in turn can help to make enduro a safe alternative to the other dangerous motorcycling sports.
KEY WORDS: sports injuries, enduro riders.

\section{Introduction}

Enduro is an off road motorcycling event. It is a fast, exciting adventure sport with increasing numbers of participants and competitions. This article reviews the published literature on physiology, risk factors and specific injuries in enduro riders.

\section{Background}

Enduro started in 1913 to test the reliability and endurance of man and machine. Although the term enduro often applies to any type of long-distance, offroad motorcycle races, its true technical definition usually refers to a set of rules, varying by the events' governing body, that specify exactly when a rider should arrive at certain pre-defined locations along a prescribed route. The object of the event is to reach those locations exactly per the defined schedule, with early or late arrivals resulting in penalties to the riders' scores. Enduro thus refers specifically to time keeping events. Races, on the other hand, are simply a course that has a start and finish, and the competitors try to race to achieve the fastest time from start to finish ${ }^{1-3}$. In this sport, if the engine breaks down, drivers have to fix it themselves. No outside help is allowed. Furthermore, driver's expertise is "examined" in difficult grounds (forests, stone ground), and their maximum speed is recorded. There are 6 members in each team and their penalty points are added. Failure of a driver to reach the defined locations can bring a team from first to last rank. As there is no option for substitution, the drivers are 'forced' to participate sometimes even if there are reasons not to (injuries, not fit, etc.). If a driver stops, that affects the points that can be earned by the team; hence the injured driver is examined by his team doctor first. Some national teams even have their own mobile $\mathrm{X}$-ray machines, so that they can quickly examine the injured driver ${ }^{1-3}$.

With the increasing popularity of this event, enduro is staged at many levels from local events through to National, Continental and World Championships ${ }^{1-3}$.

The time challenge, when added to the physical, environmental and technical difficulties which riders face on uneven and slippery courses, coupled with the pressure on the driver to keep going even if in- 
jured, makes enduro an exciting and dangerous sport. In the present article, we explore the physiological aspects of enduro riders, mechanism of injury sustained by these riders, and review the literature on various types of injuries sustained by them.

\section{Materials and methods}

A search of PubMed, Medline, CINAHL, and Embase databases was performed using the following keywords 'Enduro injuries', 'off-road motorcycle injuries' and 'Enduro sport'. Studies dealing with enduro or off road motorcycling events were identified, and their bibliographies thoroughly reviewed to identify further related articles. This search identified 4 studies which investigated the physiological characteristic of enduro riders and explored the injury pattern sustained by these athletes.

\section{Physiological aspects of enduro riders}

Scientific information on the physiological characteristic of competitive off-road motorcycle riders is scanty. Gobbi et al. ${ }^{4}$ found that enduro riders are on the upper range of the normal weight, with a mean Body Mass Index of $24.5+/-0.3$ and a body fat percentage of $12.6 \pm 3.7 \%$. This weight makes enduro riders susceptible to injuries, as it makes it more difficult to control the bike during the turns and in muddy ground, especially when going up or down hills ${ }^{4}$.

The duration of the competition and the frequent changes of direction and speed due to the characteristics of the circuit require constant involvement of all the muscle groups in the body. Therefore, both aerobic and anaerobic metabolisms are involved in enduro $^{4,5}$. Lactate levels within 2 minutes after the race are $1.5 \pm 0.3 \mathrm{mM}$, and increase to $3.8 \pm 1.3 \mathrm{mM}$ after high speed trials. Lactate accumulation leads to muscle fatigue and decreases the muscle force available for control of the motorcycle ${ }^{4,6}$.

The $\mathrm{Vo}_{2 \max }$ (maximal oxygen uptake) achieved in the upper limbs in enduro riders is between 64 and $80 \%$ of the lower limbs $\mathrm{Vo}_{2 \text { max }} 4,7$, suggesting that the work generated by the lower limbs in enduro riders was greater than the upper limbs. This could be the reason why the ankle and foot are the most common sites for severe injuries in enduro riders.

In untrained subjects, there is a significant difference in handgrip strength between the dominant and non-dominant limb. These differences reflect the greater use of the dominant arm in normal daily activities $^{8}$. In enduro riders, however, the force used for handgrip was similar in the right and left hands $\left(506 \pm 52 \mathrm{~N}\right.$ and $506 \pm 47 \mathrm{~N}$ respectively) ${ }^{4}$. Despite the similar hand grip strengths between the two hands, the occurrence of carpal tunnel like symptoms is higher on the right than the left, presumably from greater handling of the throttle, which changes the flexion angle of the wrist by the right hand ${ }^{9}$.

\section{Mechanism of injury}

The most common mechanism of injury was collision with an immobile object followed by loss of traction and collision with mobile object including other motorcycles. The rest of the injuries arose from jumping from the motorcycle, loss of control from high speed wobble, when the motorcycle starts to oscillate. In few injuries, the rider suffered from post-traumatic amnesia and was unable to recall the mechanism ${ }^{3,10}$. Two third of the injured riders were ejected from their bikes either over the handle bar $(48 \%)$ or to the right and left sides (30 and $22 \%$ respectively), while $3 \%$ were ejected backwards. The hands and arms were the most common landing points $(37 \%)$, followed by shoulders $(29.3 \%)$ then the hips and buttocks (12\%). Some of the riders, however, landed on their feet $(5 \%)$, and others on their head $(6 \%)$ or back $(2 \%)^{3,10}$. The severity of injury did not correlate with the speed at the time of accident and experience. This has been attributed to the fact that speed correlated with the amount of training and experience. Therefore, riders who were injured at higher speeds were usually highly trained seasoned participants ${ }^{10}$.

Overuse injuries have also been explored in enduro riders $^{9}$. Exposure to vibration of a motorcycle engine increased the incidence of transient carpal tunnel syndrome and forearm pain in these athletes.

\section{Anatomical distribution of injuries}

\section{A) Extremities injuries}

The extremities are the most injured parts in enduro, but $98 \%$ of these injuries are mild to moderate, with abbreviated injury scale ${ }^{10}$ grades 1 and 2 . Most of the reported injuries are ligamentous or fractures and dislocations occur with almost equal percentages (47 and $46 \%$ ). The remaining injuries were muscular strains $^{3,10}$.

\section{1) Upper Limb}

Upper limb injuries constituted $57 \%$ of the total injuries, and they involved ligamentous injuries to the shoulder girdle (eg. acromio-clavicular dislocation). Fractures and dislocations were mainly to hand and wrist composing $23 \%$ of the total fractures, followed by fractures to the humerus and clavicle. Other injuries included shoulder and wrist dislocation ${ }^{3,10}$.

The hands, the wrists and the forearms are the predominant areas of overuse in enduro riders, with symptoms suggesting carpal tunnel syndrome and transient forearm muscle tenderness ${ }^{9}$.

\section{2) Lower Limb}

Lower limb injuries accounted $43 \%$ of the total injuries to the extremities. Most of the ligamentous injuries 
were strains of the collateral ligaments of the knee. Ankle and foot were the most commonly involved areas in fractures and dislocations (36\%), with half of these fractures were to the phalanges and metatarsals. Hip dislocation has also been reported ${ }^{3,10}$.

\section{B) Head Injuries}

Injuries to the head constituted $9 \%$ of the total injuries, with concussion as the main diagnostic finding. Facial bone fractures associated with fractured dislocated mandible, broken teeth and facial laceration have also been documented ${ }^{3}, 10$.

\section{C) Abdomen and Thorax injuries}

Most of the abdominal and thoracic injuries arose from blunt trauma which resulted in bruises, broken ribs and abdominal contusions ${ }^{3}, 10$.

\section{D) Spinal injuries}

The spinal injuries accounted for $5 \%$ of the total injuries. Most cases were mild strains to the cervical and lumber spine, with no fractures or dislocations ${ }^{3,} 10$.

\section{E) Muscular injuries}

Muscular strains were reported in only $7 \%$ of injured enduro riders ${ }^{3}, 10$.

\section{Discussion}

Although motorcycling competitions are considered dangerous ${ }^{11-14}$, enduro has proved to be relatively safer, with $85 \%$ of the injuries being mild with mean Injury Severity Score ${ }^{15}$ of 3.9 , involving one anatomical area ${ }^{10}$. This could be due to the fact that the very few studies, with limited number of subjects, exist in the literature focusing on this rapidly growing sport. Keeping riders in bikes, which may be achieved by changes to the bike design, can help to decrease the severity of the injuries. Some changes to the clothing, such as provision of additional protection to the ankle and foot, which are the parts most commonly frac- tured, may be considered. Also it has been suggested that substitutes should be allowed, the number of extra tasks should be reduced, the whole area where the race takes place should be better monitored, and, in bad weather, the time limits should be wider ${ }^{3}$.

In depth understanding of the physiological aspect of enduro riders with close monitoring of injuries is needed to promote safety measures in enduro and to reduce risk factors of injury. In turn, this can help to make enduro a safe alternative to other dangerous motorcycling sports ${ }^{16}$.

\section{References}

1. http://www.acu.org.uk (accessed 10 Dec 2014)

2. http://enduro.co.uk (accessed 1 Jan 2015)

3. Schönle C. Accident causes and prevention of injuries in Enduromotorbike sport. Sportverletz Sportschaden. 1991;5(2):108-114.

4. Gobbi A, Francisco R, Kvitre R. Physiological characteristics of top level off road motorcyclists. Br J sports Med. 2005;39: 927 931.

5. Relly T, Secher N. Physiology of sports: an overview. In: Relly T, Secher N, William C, eds. Physiology of sports London: E\& FN spor. 1990;465-485.

6. Kindermann W, Kenl J. Lactate acidosis with different forms of sport activities. Can J Appl sports Aci. 1977;2:177-182.

7. Franklin BA. Exercise testing, training and arm ergometry. Sports Med. 1985;2:100-119.

8. Maughan RJ, Abel RW, Watson JS, Weir J. Forearm composition and muscle function in trained and untrained limbs. Clin Physiol. 1986;6(4):389-396.

9. Sabeti-Aschraf M, Serek M, Pachtner T, Auner K, Machinek M, Geisler M, Goll A. The Enduro motorcyclist's wrist and other overuse injuries in competitive Enduro motorcyclists: a prospective study. Scand J Med Sci Sports. 2008.

10. Colburn N, Richard M. Sports injury or trauma? Injuries of the competition off-road motorcyclist. Injury. 2003;34(3):207-214.

11. Greenspan L, Mclellan B A, Grieg H. Abbreviated injury scale and injury severity score: a score chart. J Trauma. 1985;25(1): 60-64.

12. Bachulis BL, Sangster W, Gorrell GW, Long WB. Pattern of injury in helmeted and non-helmeted motorcyclist. Am J Surg. 1988;155:708-711.

13. Bried JM, Cordasco FA, Volz RG. Medical and economic parameters of motorcycle-induced trauma. Clin Orthop. 1984; 223:252-256.

14. Deaner RM, Fitchett VH. Motorcycle trauma. J Trauma. 1975;15:678-681.

15. Baker S P, O'Neill B. The injury severity score: an update J Trauma. 1976;16:882-885.

16. Padulo J, Oliva F, Frizziero A, Maffulli N. Muscles, Ligaments and Tendons Journal. Basic principles and recommendations in clinical and field science. MLTJ. 2013; 4:250-252. 